\title{
Idosos: Associação entre o conhecimento da aids, atividade sexual e condições sociodemográficas
}

Aged people: Association between knowledge of aids, sexual activity and socialdemographic conditions

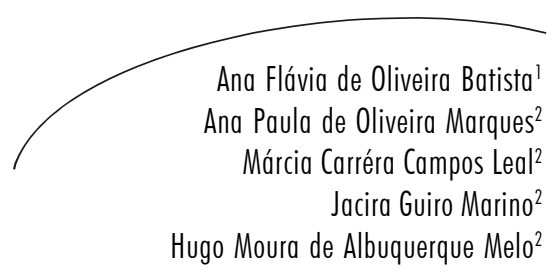

Resumo

Objetivo: Verificar a associação entre o conhecimento da Aids, atividade sexual e condições sociodemográficas em idosos participantes de programa de educação permanente de Universidade do Nordeste do Brasil. Método: Estudo transversal, realizado de março a julho de 2006, com amostra equivalente a 165 idosos, selecionados por amostragem casual simples. A coleta dos dados foi feita mediante entrevista semiestruturada. A variável dependente - conhecimento da Aids - incluiu conceito da doença, prevenção, transmissão, agente etiológico, existência de tratamento e impossibilidade de cura. Dados sociodemográficos e atividade sexual corresponderam às variáveis independentes. $\mathrm{Na}$ análise estatística utilizou-se o programa SPSS, versão 13.0, com aplicação dos testes de independência $\mathrm{X}^{2}$ de Pearson e o $\mathrm{X}^{2}$ de Kendall, adotando-se o valor de $\mathrm{p}<0,05$ para a rejeição da hipótese nula. Resultados: As respostas incompletas e incoerentes quanto ao conhecimento e transmissão corresponderam a $71,5 \%$ e $52,7 \%$, respectivamente. O uso do preservativo foi citado por $56,9 \%$ como meio de prevenção, $66 \%$ referiram existir tratamento e $66,7 \%$, ausência de cura para a doença. O agente etiológico foi identificado por $64 \%$. Quanto aos dados sociodemográficos e atividade sexual; idade 59,3\% (60-69 anos), escolaridade 72\% (mais de nove anos de estudo), estado civil $34,5 \%$ (casados), renda 45,5\% (2-4 salários mínimos), 24,8\% com atividade sexual. Verificada associação significante $(\mathrm{p}<0,01)$ entre o conhecimento da Aids e a escolaridade. Conclusões: Ainda que os resultados não possam ser extrapolados para a população idosa em geral, pode-se supor que o nível de conhecimento sobre a Aids ainda é precário, destacando-se a importância da escolaridade.

\section{Abstract}

Purpose: To verify the association between knowledge of Aids, sexual activity and social-demographic conditions in aged participants of a permanent education program at Brazil Northeast University. Method: Cross-sectional study, carried

\footnotetext{
Programa Integrado de Pós-graduação em Saúde Coletiva. Universidade Federal de Pernambuco. Recife, PE, Brasil.

2 Departamento de Medicina Social. Universidade Federal de Pernambuco, Recife, PE, Brasil.
}

Palavras-chave: Síndrome de Imunodeficiência Adquirida. Saúde do Idoso. Informação em Saúde. AIDS 
through from March to July 2006, included a sample of 165 aged people, by a simple non randomized sampling. Data collection was made with a halfstructuralized interview. Aids knowledge was the dependent variable and included concept of illness, prevention, transmission, etiological agent, existence of treatment and cure impossibility. Socio-demographic data and sexual activity corresponded to the independent variable. For statistical analysis, with SPSS software, version 13.0, Pearson independence $\mathrm{X}^{2}$ test and Kendall $\mathrm{X}^{2}$ test were performed, adopting $\mathrm{p}<0.05$ for rejection of null hypothesis. Results: Incomplete or incoherent answers of Aids knowledge and transmission corresponded to $71.5 \%$ and $52.7 \%$, respectively. Condom use for prevention was reported by $56,9 \%$, approximately $66 \%$ referred treatment existence for Aids and $66.7 \%$, absence of cure. The etiological agent was identified by $64 \%$. Socio-demographic data and sexual activity data were: age 59.3\% (60-69 years), schoolarship 72\% (more than 9 years of study), civil status 34.5\% (married), income $45.5 \%$ (2-4 minimum wages), $24.8 \%$ with sexual activity. There was significant association $(\mathrm{p}<0.01)$ between Aids knowledge and scholar level. Conclusions: Although these results cannot be generalized for the aged population, one can assume that level of knowledge on Aids is still precarious and distinguished by scholar level.
Key words: Acquired Immunodeficiency Syndrome. Aged People Health. Information on Health. Elderly's Health.

\section{INTRODUÇÃO}

Trinta e três milhões de pessoas em todo o mundo estão infectadas pelo vírus HIV, a maioria em países pobres e em desenvolvimento. $\mathrm{Na}$ América Latina, dentre os dois milhões de pessoas com HIV/aids, 27,2\% encontram-se no Brasil, sendo, portanto, o país mais afetado pela epidemia no continente americano. ${ }^{1,2}$ Seguindo uma tendência mundial, os casos de Aids têm aumentado entre os idosos, que até pouco tempo representavam apenas uma pequena parcela nas estatísticas da doença. No Brasil, 2,5\% dos idosos são portadores do HIV e esse percentual pode ser bastante superior, levando-se em conta a subnotificação de casos, além do que existe um "não-diagnóstico" neste grupo etário, resultando em altos índices de mortalidade. ${ }^{2}$

Diversos fatores são responsáveis pelo aumento $\mathrm{da}$ incidência da Aids entre a população envelhecida: aumento da utilização dos medicamentos para controle da impotência sexual; preconceito com relação à sexualidade na terceira idade; insuficiência de ações em saúde para informar aos idosos sobre a prevenção das doenças sexualmente transmissíveis e carência de conhecimento deste segmento a respeito da patologia. Em avaliação sobre as intervenções do governo brasileiro em relação à Aids, as ações para $a$ informação e prevenção da doença dirigida às pessoas idosas ainda apresentam descontinuidade. ${ }^{3,4}$

Ao assumirem a concepção social de que a velhice é assexuada, os profissionais de saúde que atendem aos idosos em geral nem sequer consideram a possibilidade da infecção pelo HIV; consequentemente, também não fornecem qualquer informação a respeito das doenças sexualmente transmissíveis e da aids. $\mathrm{O}$ tabu ainda existente em relação à sexualidade na velhice advém da concepção social de que esta é a época da vida em que o indivíduo abdica de suas atividades sexuais. A despeito da visão restrita, tanto em relação à sexualidade quanto à velhice, a exposição sexual desprotegida é atualmente a principal forma de infecção pelo HIV entre idosos. ${ }^{5}$

Pelo fato de a Aids ser cercada por tabus sexuais, a população que não pratica sexo e aquela socialmente aceita como "assexuada" costuma ser excluída das questões que envolvem as doenças sexualmente transmissíveis. Quando o idoso tem sua sexualidade socialmente negada, termina por ficar em situação de desvantagem, com relação ao 
direcionamento de campanhas preventivas sobre a Aids; e, apesar da informação sobre a transmissão e situações de risco não serem suficientes para garantir prevenção da doença, a falta de informações fidedignas contribui para aumentar a vulnerabilidade. ${ }^{6,7}$

O processo de envelhecimento, na América Latina e no Brasil, vem ocorrendo em um contexto marcado por alta taxa de pobreza, desigualdade social e desenvolvimento institucional caracterizado pela falta de sintonia com o contingente da população idosa, o que constitui um grande desafio no enfrentamento da epidemia de Aids nesse segmento etário. No Brasil, são registradas altas taxas de analfabetismo entre os idosos e a maioria tem renda per capita inferior a dois salários mínimos; a situação sociodemográfica desta população é mais alarmante quando se considera que, apesar de toda privação, quase seis milhões de idosos têm filhos e outros parentes sob sua responsabilidade. ${ }^{8,9}$

A elevação da incidência da Aids entre a população mais envelhecida é uma tendência mundial e demonstra a importância de estudos latino-americanos que analisem a situação desta epidemia na população idosa, uma vez que podem subsidiar o direcionamento de ações em saúde.

Considerando que o conhecimento a respeito da morbidade é condição para enfrentá-la e que tem ocorrido mudanças no padrão da epidemia de Aids, este estudo se propôs a verificar a associação entre conhecimento da Aids, atividade sexual e condições sociodemográficas entre idosos participantes de programa de educação permanente em Universidade do Nordeste Brasileiro.

\section{MATERIAIS E MÉTODO}

Trata-se de um estudo epidemiológico, quantitativo e transversal. Foi realizado nas instalações do Programa Universidade Aberta à Terceira Idade (UnATI), vinculado administrativamente à Pró-Reitoria de Extensão da Universidade Federal de Pernambuco (UFPE).
O Programa UnATI/UFPE, criado em setembro de 1996, é uma microuniversidade temática que aborda as questões do envelhecimento por meio de atividades de ensino, pesquisa e extensão, ou seja, cursos de extensão universitária e de atualização cultural voltados especificamente para o segmento da população com 60 anos ou mais, numa perspectiva de educação continuada. ${ }^{3}$ Tem por finalidade promover a integração universidade-idosocomunidade, envolvendo vários segmentos da comunidade em geral e da universitária, considerando a UFPE como um espaço potencial para responder às demandas sociais do envelhecimento populacional. ${ }^{10}$

A população de referência foi constituída pelas 606 pessoas, com 60 anos ou mais de idade, regularmente matriculadas nos diversos cursos oferecidos pelo programa, no período de março a julho de 2006, sendo $543(89,6 \%)$ mulheres e 63 $(10,4 \%)$ homens.

Inicialmente, em estudo piloto, envolvendo 19 idosos não alfabetizados (participantes do curso de alfabetização) e igual número de idosos alfabetizados, participantes dos demais cursos, determinou-se a proporção de pessoas com percepção aceitável sobre o que é Aids, a qual se igualou a 0,487. Para o cálculo do tamanho da amostra final, admitiram-se os parâmetros: proporção da percepção aceitável sobre o conceito de Aids igual a $0,487, z=1,96$ e efeito do desenho igual a 0,005 ( $\left.\mathrm{d}^{2}\right)$, aplicados à fórmula de Pocock, ${ }^{11}$ do que resultou amostra com 165 idosos, que não integraram o estudo piloto. A amostragem foi casual simples, mediante sorteio com uso da tabela de números aleatórios.

Foi definido "conhecimento da Aids" a noção sobre o conceito da doença, prevenção, transmissão, agente etiológico, existência de tratamento e impossibilidade de cura. As variáveis consideradas como "conhecimento" foram agrupadas em prevenção e transmissão, segundo o modelo da História Natural da Doença proposto por Leavell-Clark (1976). ${ }^{12}$

O questionário utilizado na pesquisa baseouse no protocolo utilizado no Projeto Epidoso 
(Epidemiologia do Envelhecimento), validado para a população idosa brasileira. Após a etapa piloto foram acrescentadas quatro questões no campo referente aos dados de saúde e conhecimento em relação à Aids. As variáveis independentes referentes à atividade sexual, sexo, faixa etária, estado conjugal, escolaridade e renda, foram obtidas por meio de perguntas fechadas, previamente categorizadas. Quanto ao conhecimento a respeito da Aids, foram utilizadas perguntas abertas, cujas respostas foram categorizadas por semelhança, segundo a técnica da análise de conteúdo.

No processamento estatístico dos dados foi utilizado o programa Statistical Package for Social Sciences (SPSS), versão 13.0, sendo aplicados os testes de independência $\mathrm{X}^{2}$ de Pearson e o $\mathrm{X}^{2}$ de Kendall, na análise da relação entre a variável dependente e as independentes, adotando-se o valor de $\mathrm{p}<0,05$ como nível de significância para a rejeição da hipótese nula.

O protocolo de pesquisa foi encaminhado à avaliação do Comitê de Ética em Pesquisa, do Centro de Ciências da Saúde, da Universidade Federal de Pernambuco, sendo aprovado por processo $\mathrm{n}^{\circ}$. 064/06 (CEP/CCS/UFPE). Todos os idosos participantes do estudo assinaram ou apuseram a impressão digital no Termo de Consentimento Livre Esclarecido.

\section{RESULTADOS}

As mulheres corresponderam a 91,6\% dos idosos entrevistados. A idade variou de 60 a 89 anos, com 59,3\% da amostra na faixa etária de 60 a 69 anos e 4,2\%, entre 80 e 89 anos. Quanto à escolaridade, prevaleceram idosos com nove ou mais anos de estudo (72\%), destacando-se apenas uma idosa sem escolaridade formal. Em relação ao estado conjugal, os casados ou com companheiro foram mais frequentes na amostra $(34,5 \%)$, assim como entre os homens $(71,4 \%)$, mas, entre as mulheres, a viuvez foi mais frequente $(32,4 \%)$. No que concerne à renda mensal, foi verificado que $45,5 \%$ dos idosos recebiam entre dois e quatro salários mínimos. Quando questionados sobre a atividade sexual, $73,9 \%$ dos entrevistados referiram não ter vida sexual ativa à época da entrevista. Entre os homens, 28,6\% negaram atividade sexual, percentual menor que os $78,1 \%$, entre as mulheres (tabela 1 ). 
Tabela 1 - Caracterização dos idosos entrevistados segundo variáveis sociodemográficas e atividade sexual. UnATI/UFPE . Recife, PE, 2006.

\begin{tabular}{|c|c|c|c|c|c|}
\hline \multirow{3}{*}{ Variável } & \multirow{3}{*}{ N (\%) } & \multicolumn{4}{|c|}{ Sexo } \\
\hline & & \multicolumn{2}{|c|}{ homens } & \multicolumn{2}{|c|}{ mulheres } \\
\hline & & $\mathrm{N}$ & $\%$ & $\mathrm{~N}$ & $\%$ \\
\hline Amostra & $165(100)$ & 14 & 8,4 & 151 & 91,6 \\
\hline \multicolumn{6}{|l|}{ Faixa Etária (anos) } \\
\hline 60 a 69 & $98(59,3)$ & 7 & 7,1 & 91 & 92,9 \\
\hline 70 a 79 & $60(36,5)$ & 6 & 10,0 & 54 & 90,0 \\
\hline 80 a 89 & $7(4,2)$ & 1 & 14,3 & 6 & 85,7 \\
\hline \multicolumn{6}{|l|}{ Escolaridade (anos) } \\
\hline 0 & $1(0,6)$ & - & - & 1 & 100,0 \\
\hline 1 a 4 & $23(14,0)$ & 1 & 4,3 & 22 & 95,7 \\
\hline 5 a 8 & $22(13,4)$ & 2 & 9,1 & 20 & 90,9 \\
\hline $9-12$ & $56(33,9)$ & 5 & 8,9 & 51 & 91,1 \\
\hline$>12$ & $63(38,1)$ & 6 & 9,5 & 57 & 90,5 \\
\hline \multicolumn{6}{|l|}{ Estado conjugal } \\
\hline Solteiro & $27(16,3)$ & 1 & 3,7 & 26 & 96,3 \\
\hline Casado /mora com companheiro & $57(34,5)$ & 10 & 17,5 & 47 & 82,5 \\
\hline Viúvo & $50(30,4)$ & 1 & 2,0 & 49 & 98,0 \\
\hline Separado/ divorciado & $24(14,6)$ & 2 & 8,3 & 22 & 91,7 \\
\hline Não respondeu & $7(4,2)$ & - & - & 7 & 100,0 \\
\hline \multicolumn{6}{|l|}{ Renda mensal (salários mínimos) } \\
\hline$<2$ & $44(26,6)$ & 5 & 11,4 & 39 & 88,6 \\
\hline de 2 a 4 & $75(45,5)$ & 3 & 4,0 & 72 & 96,0 \\
\hline$>4$ & $38(23,0)$ & 6 & 15,8 & 32 & 84,2 \\
\hline Não respondeu & $8(4,9)$ & - & - & 8 & 100,0 \\
\hline \multicolumn{6}{|l|}{ Atividade Sexual } \\
\hline Sim & $41(24,8)$ & 10 & 24,4 & 31 & 75,6 \\
\hline Não & $122(73,9)$ & 4 & 3,3 & 118 & 96,7 \\
\hline Não respondeu & $2(1,3)$ & - & - & 2 & 100,0 \\
\hline
\end{tabular}

No tocante ao conhecimento sobre Aids, $6,7 \%$ dos idosos conceituaram-na corretamente como "doença que afeta o sistema imunológico"; $21,8 \%$ não atribuíram qualquer conceito para a doença e 71,5\% a definiram de forma incompleta. Com relação às formas de transmissão, a informação mais abrangente "contato sexual, transfusão de sangue, uso de drogas injetáveis e de material não estéril" foi fornecida por $47,3 \%$ dos idosos e as respostas ausentes ou incompletas somaram $52,7 \%$. Quanto à prevenção, a resposta "usar preservativo, evitar múltiplos parceiros, não compartilhar seringas”, considerada mais completa segundo a literatura, foi fornecida por $15,8 \%$ dos idosos; o uso do preservativo foi a referência mais citada (57\%) e a ausência de informação referida somada a respostas do tipo "ser responsável, usar anticoncepcional, evitar contato com aidéticos, ir ao médico fazer prevenção, evitar contato sexual", consideradas em conjunto como "outras formas", totalizou $27,2 \%$. O vírus HIV foi reconhecido como agente etiológico da Aids por $63,7 \%$ dos idosos; contudo, $36,3 \%$ não forneceram qualquer resposta ou informaram de maneira incorreta. A maior parte da população investigada demonstrou ter informações corretas 
sobre a existência de tratamento e impossibilidade de cura para a Aids (tabela 2). Os testes estatísticos demonstraram associação significante entre $\mathrm{O}$ conhecimento da Aids (conceito da doença e identificação do agente etiológico) e escolaridade $(\mathrm{p}<0,01)$ (tabela 3).

Tabela 2 - Identificação do conhecimento sobre Aids. UnATI/UFPE. Recife, PE, 2006.

\begin{tabular}{|c|c|c|}
\hline Conhecimento referido sobre Aids & $\mathrm{N}$ & $\%$ \\
\hline \multicolumn{3}{|l|}{ O que é Aids? } \\
\hline Doença que afeta o sistema imunológico & 11 & 6,7 \\
\hline Doença sexualmente transmissível de tratamento difícil & 31 & 18,8 \\
\hline Doença incurável, infecciosa, transmitida por sexo e sangue & 32 & 19,4 \\
\hline Doença de quem não sabe se prevenir, de homossexual, fruto do pecado & 30 & 18,2 \\
\hline Doença causada por vírus ou germe & 25 & 15,1 \\
\hline Não respondeu & 36 & 21,8 \\
\hline \multicolumn{3}{|l|}{ Como se pega Aids? } \\
\hline $\begin{array}{l}\text { Contato sexual/Transfusão de sangue/Uso de drogas injetáveis/Uso de material não } \\
\text { estéril }\end{array}$ & 78 & 47,3 \\
\hline Contato sexual & 61 & 37,0 \\
\hline Contato sexual/Beijo & 3 & 1,8 \\
\hline Não respondeu & 23 & 13,9 \\
\hline \multicolumn{3}{|l|}{ O que faz ou faria para se prevenir da Aids? } \\
\hline Usar preservativo/Evitar múltiplos parceiros/Não compartilhar seringas & 26 & 15,8 \\
\hline Usar preservativo & 94 & 57,0 \\
\hline Outras formas & 24 & 14,5 \\
\hline Não respondeu & 21 & 12,7 \\
\hline \multicolumn{3}{|l|}{ Qual é o vírus da Aids? } \\
\hline Respondeu de forma correta (HIV) & 105 & 63,7 \\
\hline Respondeu de forma incorreta & 6 & 3,6 \\
\hline Não respondeu & 54 & 32,7 \\
\hline \multicolumn{3}{|l|}{ Existe tratamento para a Aids? } \\
\hline Sim & 109 & 66,1 \\
\hline Não & 41 & 24,8 \\
\hline Não sabe & 14 & 8,5 \\
\hline Não respondeu & 1 & 0,6 \\
\hline \multicolumn{3}{|l|}{ A Aids tem cura? } \\
\hline Não & 110 & 66,7 \\
\hline Sim & 33 & 20,0 \\
\hline Não sabe & 16 & 9,7 \\
\hline Não respondeu & 6 & 3,6 \\
\hline
\end{tabular}


Tabela 3 - Correlações entre as variáveis dependente e independentes. UnATI/UFPE. Recife, PE, 2006.

\begin{tabular}{llc}
\hline Variáveis independentes & Variável dependente & Correlação \\
\hline Sexo & Conhecimento sobre a Aids & $\mathrm{p}>0,05^{\mathrm{c}}$ \\
Faixa Etária (anos) & Conhecimento sobre a Aids & $\mathrm{p}>0,05^{\mathrm{c}}$ \\
Estado conjugal & Conhecimento sobre a Aids & $\mathrm{p}>0,05^{\mathrm{c}}$ \\
Escolaridade (anos) & Conceito da Aids & $\mathrm{p}<0,01^{\mathrm{a}}$ \\
Renda mensal & Identificação do agente etiológico & $\mathrm{p}<0,01^{\mathrm{b}}$ \\
Prática sexual & Conhecimento sobre a Aids & $\mathrm{p}>0,05^{\mathrm{c}}$ \\
\hline
\end{tabular}

a $-\mathrm{p}<0,01$ (Correlação de Pearson)

b - p $<0,01$ (Correlação de Kendall)

c - p > 0,05 (Correlação de Pearson)

\section{DISCUSSÃO}

A predominância de idosos do sexo feminino, concentrada na faixa de idade de 60 a 69 anos, pareceu refletir a distribuição brasileira de idosos. As mulheres predominam em todas as faixas etárias, sendo também maioria entre os alunos matriculados em programas de educação permanente. ${ }^{15}$

A Pesquisa Nacional de Amostra de Domicílios (PNAD), em 2001, indicava que 50\% dos indivíduos com 60 anos ou mais de idade eram analfabetos. Em 2008, essa pesquisa permitiu comprovar que as pessoas com 60 anos ou mais de idade, do Nordeste, tinham em média 2,7 anos de estudo, denotando que nossa amostra diferiu desse contexto, já que $85,4 \%$ tinham mais de quatro anos de estudo. ${ }^{16}$ Esse achado pode ser uma característica diferencial derivada da seleção amostral. Os idosos eram participantes das atividades da Universidade Aberta à Terceira Idade, daí terem nível educacional maior. ${ }^{10} \mathrm{O}$ nível educacional interfere diretamente no desenvolvimento da consciência sanitária, na capacidade de entendimento do tratamento prescrito e na prática do autocuidado, com relevância considerável quando da estruturação de campanhas educativas. ${ }^{17,18}$

A distribuição da escolaridade pareceu ter influenciado também a renda mensal, confirmada pela maioria dos idosos investigados, maior que a relatada na PNAD de $2008 .{ }^{16} \mathrm{~A}$ escolaridade é um fator que contribui para a manutenção da atividade econômica, porque pode agir compensando a perda da capacidade laborativa. ${ }^{18}$ No entanto, a presente pesquisa permitiu também identificar a perda de poder aquisitivo desses idosos, já que houve uma redução de renda quando comparada a estudo realizado em 2003, envolvendo 308 idosas do mesmo grupo. ${ }^{10}$

Pode-se aventar a hipótese de ter havido maior divulgação do programa da UnATI, ao longo do tempo, atraindo uma população de idosos mais heterogênea, com menor escolarização e, consequentemente, menor renda. Isso encontra respaldo na redução do número de anos de estudo do grupo da presente pesquisa, quando comparado à realizada em 2003, na qual $75,5 \%$ apresentavam segundo grau completo ou nível superior, percentual que se reduziu para $72 \%$ na presente pesquisa. ${ }^{10}$

Pesquisa investigando o impacto do arranjo familiar na saúde dos idosos brasileiros constatou que a grande maioria dos homens era casada e morava com o cônjuge, no que diferia das mulheres. Estas tinham mais dificuldade para casar novamente após ficarem viúvas, o que pode explicar a maior quantidade de idosas sem companheiro, ${ }^{19}$ também identificada neste estudo, e referida por Barreto et al.. ${ }^{10}$

Corroborando os dados do presente estudo, com relação à atividade sexual, pesquisa realizada 
pelo Instituto Brasileiro de Opinião Pública e Estatística (IBOPE) constatou que $55 \%$ dos adultos com idade de 55 anos ou mais negaram atividade sexual. ${ }^{20}$ Com o avanço da idade, é possível que a atividade sexual diminua, sobretudo em função do declínio das condições físicas. Mesmo assim, não se pode afirmar que os idosos não praticam sexo por desinteresse. $O$ fato de não terem parceiro fixo pode ser uma explicação mais plausível para os idosos negarem a atividade sexual. ${ }^{4}$

Com o advento dos medicamentos para a disfunção erétil, a frequência de exposições sexuais entre os idosos tem aumentado. O contato sexual é a principal forma de transmissão da Aids entre a população com 60 anos ou mais de idade. .,21,22 $^{2}$ Os resultados apresentados denotam que o grupo investigado não tinha conhecimento adequado sobre o conceito da Aids, tal como identificado em estudo brasileiro, realizado em 2001, envolvendo 120 idosos, dos quais $53,5 \%$ a definiram como "doença contagiosa" e 14\% declararam desconhecê-lo. ${ }^{7}$ Esses dados são preocupantes porque indicam que a realidade não se modificou ao longo dos anos.

Os idosos da presente pesquisa demonstraram ter conhecimento correto sobre a transmissão sexual da Aids, mas não responderam adequadamente quanto à totalidade dos meios de transmissão da doença. Estudo realizado nos Estados Unidos da América identificou que, dentre 514 idosos, 85\% tinham informações insuficientes sobre a transmissão sexual da aids ${ }^{21}$. Também nos Estados Unidos, pesquisa realizada por Zablotsky \& Kennedy, ${ }^{23}$ quanto ao conhecimento sobre a transmissão e atitudes de risco para contrair a Aids expresso por 745 idosos, identificou a utilização de seringas compartilhadas como o principal meio de transmissão citado por 98\% dos respondentes. Tais achados estão de acordo com os resultados do atual estudo.

Embora o preservativo seja a forma de prevenção mais conhecida pelos idosos, é seis vezes menos utilizada do que entre a população mais jovem. ${ }^{4} \mathrm{O}$ uso do preservativo é extremamente problemático para as mulheres na menopausa, sem nenhum risco de engravidar, por acreditarem que não precisam de proteção. Dentre os homens, ressalta-se o preconceito quanto ao uso do preservativo. ${ }^{5,24} \mathrm{~A}$ identificação positiva do HIV como agente etiológico pela maioria dos idosos demonstrou conhecimento correto sobre o agente causador da doença, mas cabe destacar que porcentagem significativa da amostra respondeu incorretamente ou não informou. A vulnerabilidade social e pessoal a que está exposto o idoso para contrair o HIV aumenta com a carência de informações precisas, relevantes e abrangentes sobre o agente etiológico da Aids e sobre as formas de transmissão do vírus. ${ }^{21,23-26}$

Ainda que a maioria dos idosos tenha respondido corretamente sobre a existência de tratamento para a Aids, parcela significativa não forneceu informações corretas sobre a questão. Embora o conhecimento dos idosos sobre a Aids esteja aumentando em alguns aspectos, incluindo os fatores de risco para a transmissão da doença, esta população ainda se encontra desinformada sobre o próprio risco para contrair a enfermidade e também em relação ao tratamento. ${ }^{23,27}$

Reconhecer as formas de prevenção e transmissão da Aids é importante tanto para os jovens quanto para os idosos. Para estes, há uma relevância ainda maior, uma vez que a falta de informação sobre a doença pode resultar no contágio pelo vírus HIV e a demora no diagnóstico e no tratamento podem culminar com óbito em menor tempo, em relação a uma pessoa mais jovem..$^{21,23,26}$

Considerando o percentual de omissões às perguntas e de respostas incompletas, constatouse ter sido precário o conhecimento dos idosos sobre a aids. Mesmo não podendo extrapolar os resultados da atual pesquisa para a população em geral, pode-se ao menos supor que, se em uma amostra diferenciada quanto a escolaridade e renda, o conhecimento sobre a aids ainda é insuficiente, a realidade deve ser bem mais sombria, quando se considera a maioria dos idosos brasileiros.

A presente pesquisa não demonstrou associação entre o conhecimento da aids e as 
variáveis sexo, faixa etária, estado conjugal, renda e atividade sexual. Tais achados diferem de estudo no qual o conhecimento se mostrou associado de forma inversa à idade e de forma direta à renda. ${ }^{4}$ Pesquisas realizadas por Henderson et al. ${ }^{21} \mathrm{e}$ Zablotsky \& Kennedy ${ }^{23}$ também não encontraram associação significante entre o conhecimento referido sobre a Aids e o estado conjugal, sendo concordante com os resultados ora apresentados. Ainda com relação ao estado conjugal, investigação realizada por Neundorfer et al. ${ }^{25}$ sobre os fatores de risco para contrair a Aids relatados por idosas verificou que as divorciadas ou separadas tinham maior conhecimento sobre a Aids do que as casadas ou solteiras.

A associação estatisticamente significante entre escolaridade e conhecimento sobre a Aids $(p<0,01)$, observada neste estudo, pode estar relacionada com a tendência atual da epidemia, acometendo prioritariamente a população com menor escolaridade. Inelmen et $a .^{5}$ destacam relação inversa entre os anos de estudo dos idosos e a incidência da Aids. Henderson et al., ${ }^{21}$ investigando a associação entre as variáveis sociodemográficas e o conhecimento de idosos a respeito da Aids, identificaram correlação diretamente proporcional entre os anos de estudo

\section{REFERÊNCIAS}

1. UNAIDS. Global summary of the AIDS epidemic. 2006 ; Disponível em: < http:// www.unaids.org/en/HIV_data/ 2006GlobalReport/defaut.asp > . Acesso em: 26/ 12/2006.

2. Brasil. Ministério da Saúde. Serviço de Vigilância em Saúde. Departamento de DST/ AIDS e Hepatites Virais. Boletim Epidemiológico Aids - DST, versão preliminar 2009. Brasília: MS/SVS/ Departamento de DST/ AIDS e Hepatites Virais 2009, 23 p. Disponível em: www.aids.gov.br/data/documents/.../ Boletim2009_preliminar.pdf. Acesso em 25/02/ 2010.

3. Gomes SF, Silva CM. Perfil dos idosos infectados pelo HIV/Aids: uma revisão. Vittale 2008;20(1):107-122.

4. Gott C. Sexual activity and risk-taking in later life. Health Soc Care 2001;9(2):72-78. dos indivíduos e o conhecimento em relação à doença. Mesmo diante dessas evidências, é necessário cautela ao analisar os resultados $\mathrm{da}$ relação entre escolaridade e conhecimento sobre a Aids, porque a baixa escolaridade pode contribuir para uma pobre compreensão da doença, ainda que o indivíduo receba informações

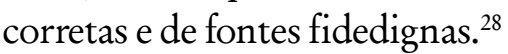

\section{CONCLUSÃO}

No Brasil, grandes avanços já foram alcançados com relação à assistência, principalmente no tocante à legalização dos direitos da pessoa idosa. No entanto, o segmento idoso ainda não é priorizado. As ações de saúde dirigidas aos mais velhos relacionadas à Aids só recentemente foram foco de campanha, mas ainda estão diluídas na assistência a outros grupos etários. É urgente que os órgãos competentes, nas três esferas de governo, elaborem ações específicas voltadas aos idosos, tomando-se como base o avanço da epidemia nesse segmento. Ainda que os resultados não possam ser extrapolados para a população idosa em geral, pode-se supor que o nível de conhecimento sobre a Aids ainda é precário, destacando-se a importância da escolaridade.
5. Inelmen E, et al. HIV/AIDS in older adults: a case report and literature review. Geriatrics 2005;60(9):56-63.

6. Lindau ST, et al. A study of sexuality and health among older adults in the United States. N Engl J Med 2007;357(8):762-74.

7. Vasconcelos E, et al. Perfil epidemiológico dos clientes HIV/AIDS na terceira idade. Rev Bras Enferm, Brasília 2001;54(3):435-445.

8. Indicador de Alfabetismo Funcional( Brasil). Indicador de alfabetismo funcional principais resultados: Ação do Ibope pela Educação. Instituto Paulo Montenegro 2009. 18.

9. Martin $\mathrm{G}$, et al. Assistência hospitalar à população idosa em cidade do sul do Brasil. Epidemiol Serv Saude 2006;15(1):59-65.

10. Barreto KML, Carvalho EMF, Falcão IV, Lessa FJD, Leite VMM. Demographic social- 
epidemiological profile of elderly women enrolled at the open University Program for Senior Citizens in the State of Pernambuco. Rev Bras Saude Matern Infant 2003;3(3).339-354.

11. Pocock SJ. Clinical trials. A practical approach. Chichester: John Wiley, 1996.

12. Scliar M. História do Conceito de Saúde. Physis 2007; 17(1):29-41.

13. Medeiros K. Avaliação do nível de informação em relação à Aids por idosos da comunidade 27 de novembro . Recife. Monografia (Especialização em Geriatria e Gerontologia) Universidade Estácio de Sá; 2005.

14. Vieira E, et al. Alguns aspectos do comportamento sexual e prática de sexo seguro em homens do município de São Paulo. Cad Saude Publica 2000;16(4):977-1009.

15. Alves LC, et al. Fatores associados à incapacidade funcional dos idosos no Brasil: análise multinível. Rev Saúde Pública 2010;44(3): 1-11.

16. Brasil. Ministério do Planejamento, Orçamento e Gestão. Instituto Brasileiro de Geografia e Estatística - IBGE. Pesquisa Nacional por Amostral de Domicílios (PNAD). Rio de Janeiro;2008;29:1-129.

17. Reis LA, et al. Características sóciodemográficas e de saúde de idosos de uma instituição do município de Jequié/BA. Rev Espaço para a Saúde 2008; 9(2): 39-46

18. Pérez ER. Saúde e Trabalho dos Idosos em São Paulo: um estudo através da SABE. Belo Horizonte. [Dissertação] ,Universidade Federal de Minas Gerais; 2005.
19. Almeida LA, Patriota LM. Sexualidade na terceira idade: um estudo com idosas usuárias do programa saúde da família do bairro das cidades - Campina Grande/PB. Qualitas 2009: 8(1): 1-20

20. Paiva V, et al. Uso de preservativos .Pesquisa Nacional MS / Ibope; 2003. [Acesso em: 11. nov 2006] Disponível em: http://www.aids.gov.br.

21. Henderson S, et al. Older women and HIV: How much do they know and where are they getting their information? J Am Geriatr Soc 2004;52(9):1549-1553.

22. Sousa JL. Sexuality in old age: a discussion about aids aging and medicines for erectile dysfunction. DST 2008;20(1):59-64.

23. Zablotsky D, Kennedy M. Risk factors and HIV transmission and older women: knowledge, options and the initiation of safer sexual practices. J Acquir Immune Defic Syndr 2003;33:122-130.

24. Mack A, Ory G. Aids and older Americans at the end of the twentieth century. J Acq Imm Defic Syndr 2003;33:68-75.

25. Neundorfer M, et al. HIV-risk factors for midlife and older women. Gerontol 2005;45:617-625.

26. Risman A. Sexualidade e terceira idade: uma visão histórico-cultural. Textos sobre Envelhecimento 2005;8(1):15-27.

27. Wolff A, Selik R. Epidemiology of acquired immune deficiency syndrome in persons aged 50 years or older. J Acquir Immune Defic Syndr 1991;33(1):84-88.

28. Wong LLR, Carvalho JA. O rápido processo de envelhecimento populacional do Brasil: sérios desafios para as políticas públicas. Rev. bras. Est Pop 2006; 23(1):5-26. 